Clinical Reports

\title{
Rickettsial infection presenting as culture-negative meningitis
}

\author{
M.L. Woo, ${ }^{1}$ J.W.C. Leung ${ }^{2}$ and G.L. French ${ }^{1}$ \\ Departments of ${ }^{1}$ Microbiology and ${ }^{2}$ Medicine, The Chinese University of Hong Kong, Prince of Wales \\ Hospital, Shatin, N.T., Hong Kong.
}

\begin{abstract}
Summary: Meningitis is an unusual presentation of rickettsial infection. We report a patient who was confirmed by serology to have typhus fever (not scrub), but who presented with the typical findings of culture-negative bacterial meningitis. Neurological manifestations of different types of rickettsial infection are discussed.
\end{abstract}

\section{Introduction}

Scrub typhus is the most common form of rickettsial infection in Hong Kong, although other members of the typhus group are occasionally seen. ${ }^{1}$ Twelve cases of scrub typhus and one of other typhus (urban) were reported to the Hong Kong Medical and Health Department in $1985^{1}$ but the incidence of rickettsial infection is probably much higher than this ${ }^{2}$ and many patients with unusual presentations go undiagnosed. In 1987, Austin et al. reported a case of culture-negative endocarditis in a patient with serological evidence of rickettsial (murine typhus) infection. ${ }^{3}$ In 1986, we saw an unusual case in Hong Kong presenting as culture-negative meningitis.

\section{Case report}

A 58 year old coppersmith was admitted to the Prince of Wales Hospital because of severe headaches for 3 days and repeated vomiting. On admission, he was conscious but very dull. There was marked neck rigidity. No focal neurological deficit was detected. There was no skin rash. Blood culture was taken and was later found to be sterile. The patient was afebrile on admission but 2 days later had a fever of $38.8^{\circ} \mathrm{C}$. The peripheral white cell count was $32.8 \times 10^{9} / 1$. Lumbar puncture was performed and turbid cerebrospinal fluid (CSF) was obtained. The results of CSF examination are shown in Table I. Gram stain of the CSF revealed no organisms but because of the high CSF white cell count, high protein and low glucose concentration, bacterial

Correspondence: M.L. Woo

Accepted: 8 March 1988 meningitis was suspected. Antigens of Streptococcus pneumoniae, Haemophilus influenza $\mathrm{b}$ and Neisseria meningitidis were not detected by latex agglutination (Phadebact CSF test, Pharmacia Diagnostics AB, Uppsala, Sweden; Slidex meningite-kit, bioMérieux Laboratory reagents and products, France), but the clinical presumptive diagnosis was meningococcal meningitis. The patient was therefore treated with penicillin $\mathbf{G}$ and chloramphenicol intravenously. Four days after admission his general condition had markedly improved and by day 7 the fever had subsided. Throat cultures were repeatedly negative. The peripheral white cell count had fallen to $12.9 \times 10^{9} / 1$ by day 10 .

Chloramphenicol therapy was stopped on day 10 , and the following day the patient developed a remittent fever. Investigations were repeated, including cultures of sputum, throat, urine and blood, and serology for brucella and leptospira. Lumbar puncture was repeated on day 13 (Table I). This CSF sample was also sent for mycobacterial investigation. Bone marrow was aspirated for culture and histology. The results of all these investigations were negative. On day 18 , because of the persistent swinging fever, cefuroxime and gentamicin were added to the penicillin treatment.

On day 21, blood was taken for the Weil-Felix test and the results were available on day 23 (Table II). Since the titre of OX19 was 320 , rickettsial infection was suspected. The previous antimicrobial therapy was stopped and replaced with oral tetracycline. After 4 days of tetracycline, the fever resolved and the patient became well. He was discharged on day 33 with a 10-day supply of tetracycline, and has since remained well. Outpatient follow-up revealed a four-fold drop in the

(C) The Fellowship of Postgraduate Medicine, 1988 
Table I Results of cerebrospinal fluid examination

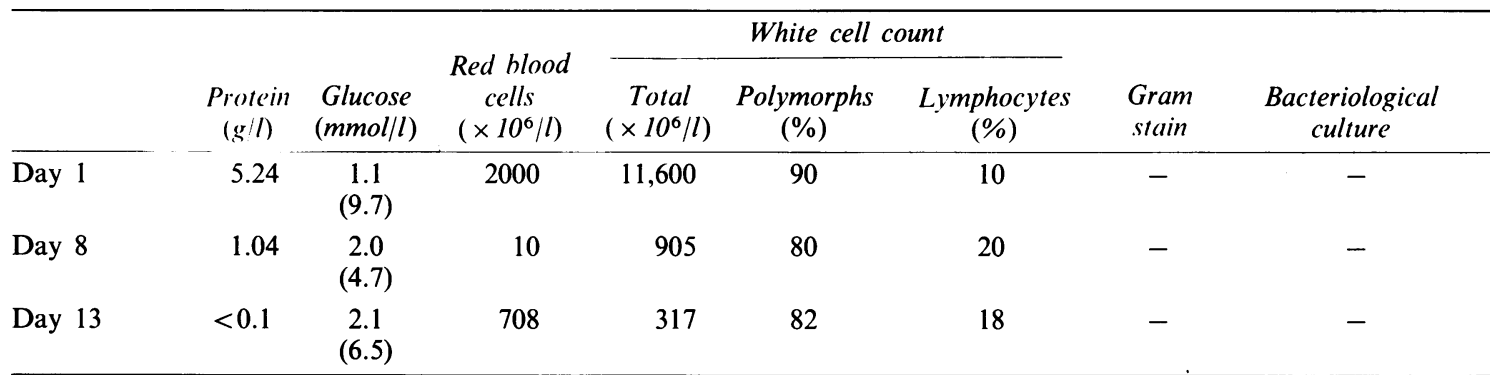

Figures in parentheses indicate blood glucose levels taken simultaneously; - denotes negative result.

Table II Results of Weil-Felix reaction

\begin{tabular}{lrrr}
\hline \multicolumn{1}{c}{ Time } & $O X 19$ & $O X 2$ & $O X K$ \\
\hline Day 21 & 320 & 20 & $<20$ \\
6 weeks & 320 & 40 & $<20$ \\
3 months & 80 & 20 & $<20$ \\
4 months & 80 & $<20$ & $<20$ \\
\hline
\end{tabular}

titre of OX19 after 2 months and a similar fall in the OX2 titre after 3 months (Table II). The diagnosis of rickettsial infection, probably typhus fever other than scrub typhus, was confirmed by clinical response to chloramphenicol and tetracycline, and a four-fold fall in antibody titres in the Weil-Felix reaction.

\section{Discussion}

This patient had a typical clinical presentation of bacterial meningitis - headache, vomiting, fever, drowsiness and neck rigidity. He also had the typical CSF changes of bacterial meningitis, including turbidity, high protein and low glucose concentrations, and a high white cell count with predominant polymorphs. He was therefore treated initially as a case of bacterial meningitis, and responded to therapy which included chloramphenicol. However, special investigations failed to confirm a bacterial infection, and when the chloramphenicol was stopped, the fever returned. There was no response to further treatment with penicillin, cefuroxime and gentamicin. A Weil-Felix test suggested the diagnosis of rickettsial infection, and the patient responded to oral tetracycline alone.

This patient is more likely to have had typhus fever than spotted fever, since the latter disease is rare in the Far East ${ }^{4,5}$ and usually shows a high anti-OX2 titre of about 1:160 in the third week. ${ }^{4}$ $\mathrm{He}$ is unlikely to have had scrub typhus since the
anti-OXK titre was never raised. However, it is impossible to differentiate between murine and epidemic typhus on the basis of the Weil-Felix reaction. ${ }^{6}$ This can be done by a complement-fixation test $^{4,6}$ but this was not available. Murine typhus is the more likely diagnosis since this form is endemic in most countries of South East Asia ${ }^{7}$ while epidemic typhus is uncommon and limited to highland areas. ${ }^{8}$ Serological surveys of human and rat populations in tropical and subtropical urban centres suggest that murine typhus occurs more frequently than is generally recognized and its importance as a human disease is probably grossly underestimated. ${ }^{8}$

Neurological manifestations of murine typhus include headache, stupor, prostration, delirium, agitation, coma, neck rigidity and general spasticity. ${ }^{4,6}$ These symptoms may suggest meningitis, but the CSF is usually normal except for slight increases in pressure and lymphocytes. ${ }^{4}$ Neurological features of epidemic typhus range from headache and general spasticity to extreme agitation, stupor and coma. ${ }^{4}$ Rocky Mountain spotted fever may present with headache, back stiffness, muscular rigidity, convulsions and coma, but the CSF is reported to be normal. ${ }^{4}$ Harrell $^{9}$ studied 35 patients with spotted fever and reported that red blood cells were often found in the CSF. He also found that the cellular reaction was usually lymphocytic with a concentration of about $10 \times 10^{6} / 1$. In only two of his cases was the cell count greater than $50 \times 10^{6} / 1$. Spinal fluid protein concentration was usually about $1 \mathrm{~g} / \mathrm{l}$, and glucose and chloride concentrations were all normal. Harrell also reported that the greatest degree of involvement of the central nervous system occurs in spotted fever, followed by epidemic typhus and scrub typhus.

Thus, it is well recognized that patients with typhus fever may present with a clinical picture of encephalitis or meningoencephalitis but to our knowledge, this is the first reported case of rickettsial infection presenting as meningitis. Since chloramphenicol is often used for the treatment of acute 
meningitis in South East Asia, it is possible that this presentation is more common than reports would indicate. Serological tests for rickettsial

\section{References}

1. Thong, K.L. (Director of Medical and Health Services). Hong Kong Annual Departmental Report 1985-1986. Hong Kong Government, Hong Kong, 1986: Statistical tables 22.

2. French, G.L., Vallance-Owen, J. \& Woo, M.L. Scrub typhus at the Chinese University of Hong Kong. $J$ Hong Kong Med Assoc, 1985, 37: 191-192.

3. Austin, S.M., Smith, S.M., Co, B., Coppel, I.G. \& Johnson, J.E. Case report: serologic evidence of acute murine typhus infection in a patient with culturenegative endocarditis. Am J Med Sci 1987, 293: 320-323.

4. Woodward, T.E. Rickettsial infections. In: Petersdorf. R.G., Adams, R.D., Braunwald, E., Isselbacher, K.J.. Martin, J.B. \& Wilson, J.D. (eds) Harrison's Principles of Internal Medicine. McGraw-Hill, New York, 1983. pp. 1066-1078. infection should be performed on patients with culture-negative meningitis who present in endemic areas.

5. Uchida, T., Mahara, F., Tsuboi, Y. \& Oya, A. Spotted fever group rickettsiosis in Japan. Jpn J Med Sci Biol 1985, 38: 151-153.

6.Ormsbee, R.A. Rickettsiae. In: Lennette, E.H., Balows, A., Hausler, W.J. \& Shadomy, H.J. (eds) Manual of Clinical Microbiology. American Society for Microbiology, Washington, 1985, pp. 845-855.

7. Arthopod- and rodent-borne viral and rickettsial diseases in the WHO South-East Asia and Western Pacific Regions: Memorandum from a WHO Meeting. Bull WHO 1983, 61: 435-436.

8. WHO working group on rickettsial diseases. Rickettsioses: A continuing disease problem. Bull WHO 1982, 60: $157-164$.

9. Harrell, G.T. Rickettsial involvement of the nervous system. Med Clin North Am 1953, 37: 395-422. 